\title{
Microwave Spectrum, Nuclear Quadrupole Coupling, and Structure Dependent Parameters of 2-Chlorobuten-3-yne
}

\author{
FRED KARLSSON, MATS GRANBERG and RAGNAR VESTIN
}

Department of Physical Chemistry, Arrhenius Laboratory, University of Stockholm, S-104 05 Stockholm 50, Sweden

The microwave spectra of the two natural species of 2-chlorobuten-3-yne, $\mathrm{CH}_{2} \mathrm{C}^{35} \mathrm{ClCCH}$ and $\mathrm{CH}_{2} \mathrm{C}^{37} \mathrm{ClCCH}$, have been measured in the region $27000-40000 \mathrm{MHz}$. The rotational constants $A, B$ and $C$ for the ground state as well as the centrifugal distortion constants $\Delta_{\mathrm{J}}, \Delta_{\mathrm{JK}}, \Delta_{\mathrm{K}}, \delta_{\mathrm{J}}$, and $\delta_{\mathrm{K}}$ were determined. From the hyperfine splittings of the rotational lines, the nuclear quadrupole coupling constants $\chi_{\mathrm{aa}}$ and $\chi_{\mathrm{bb}}$ were calculated for the $\mathrm{CH}_{2} \mathrm{C}^{58} \mathrm{ClCCH}$ and $\mathrm{CH}_{2} \mathrm{C}^{37} \mathrm{ClCCH}$ species, in the principal-axis system of the molecules.

In order to supply complementary data for electron diffraction measurements, the inertial moments corresponding to the average structure were calculated from an estimated force field. The obtained values are $I_{\mathrm{a}}{ }^{2}=73.634, I_{\mathrm{b}}{ }^{2}=$ 163.405 and $I_{\mathrm{c}} \mathrm{z}^{\mathrm{z}}=237.054$ au $\AA^{2}$ for $\mathrm{CH}_{2} \mathrm{C}^{35}$. ClCCH and $I_{\mathrm{a}}{ }^{2}=74.393, \quad I_{\mathrm{b}}{ }^{2}=166.740$ and $I_{\mathrm{c}}{ }^{2}=241.149 \mathrm{au} \AA^{2}$ for $\mathrm{CH}_{2} \mathrm{C}^{87} \mathrm{ClCCH}$.

For some time we have been concerned with the study of the structure of the three isomeric chlorobutenynes, trans-1-chlorobuten-3-yne, cis1-chlorobuten-3-yne, and 2-chlorobuten-3-yne, by electron diffraction in the gas phase (in cooperation with the electron diffraction group at Oslo University).

In order to supply additional structural information we have started to investigate the three chlorobutenynes by microwave spectroscopy. 1

The primary structure dependent parameters obtained by microwave spectroscopy are the principal moments of inertia. These are related to the spectroscopic effective structure $r_{0}$ which is a different structure to that obtained by electron diffraction in gases due to the internal vibrations. $^{2}$ However, it is possible in both methods to obtain the average structure if the harmonic potentials are known.,4 These corrections of the microwave spectroscopic parameters have been made for a simple estimated diagonal force field.

The centrifugal distortion parameters have also been calculated and compared with those obtained from the measured microwave spectrum to test the validity of the estimated force field. The value of these corrections is, of course, very limited due to the uncertainty in the estimated force field and the approximations used. However, the magnitudes of the corrections form the $r_{0}$ to $r_{z}$-parameters are interesting in themselves and may be used to see how the inertial moments are affected.

\section{EXPERIMENTAL}

According to the method of Nieuwland et al. ${ }^{5}$ acetylene is dimerized in a liquid $\mathrm{Cu}(\mathrm{I})$ catalyst in a continuous process:

$$
2 \mathrm{HC} \equiv \mathrm{CH} \rightarrow \mathrm{CH}_{2}=\mathrm{CH}-\mathrm{C} \equiv \mathrm{CH}
$$

If a small flow of $\mathrm{O}_{2}$ and $\mathrm{HCl}$ is introduced with the entering gas a small amount of chlorohydrocarbon forms: ${ }^{6,7}$

$$
2 \mathrm{C}_{2} \mathrm{H}_{2}+\frac{1}{2} \mathrm{O}_{2}+\mathrm{HCl} \rightarrow \mathrm{C}_{4} \mathrm{H}_{3} \mathrm{Cl}+\mathrm{H}_{2} \mathrm{O}
$$

We used a sample from this reaction and the substance was isolated and purified with gas liquid chromatography at a temperature of $50^{\circ} \mathrm{C}$. The column was packed with diethylhexyl-sebacate (15\%) absorbed on Chromosorb.

The product was originally identified as 2 chlorobuten-3-yne with IR, NMR, and mass spectroscopy. ${ }^{7}$ The microwave spectroscopic investigation confirms this result.

The microwave spectra were recorded on a Hewlett-Packard model 8460A R-band 


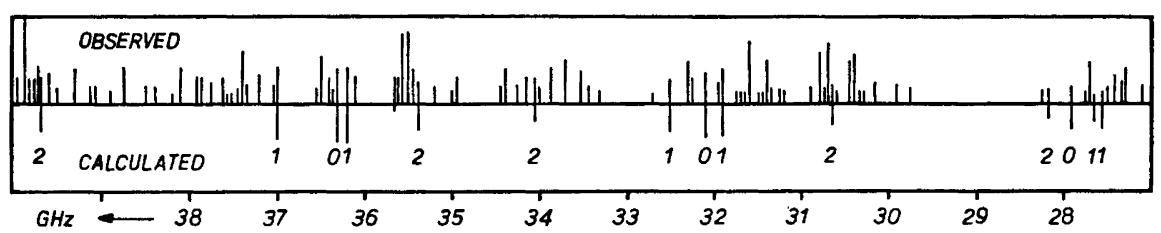

Fig. 1. The observed microwave transitions from a fast sweep ( $1 \mathrm{MHz} / \mathrm{sec})$ in the region $27-40$ GHz together with the expected strongest $R_{\mathrm{a}}$-transitions $K_{-1}=0 \rightarrow 0, K_{-1}=1 \rightarrow 1$ and $K_{-1}=2 \rightarrow 2$ with their relative intensities.

microwave spectrometer with a phase stabilized source oscillator. The recordings were made at room temperature, with the pressure ranging from 5-20 mTorr. The frequency region was $27000-40000 \mathrm{MHz}$. The precision of the measured transitions was estimated to be 0.05 MHz.

\section{MICROWAVE SPECTRUM}

In order to assign the spectrum the region $27000-40000 \mathrm{MHz}$ was swept at a rate of $1 \mathrm{MHz} / \mathrm{s}$. The observed transitions were plotted on a $50 \mathrm{MHz} / \mathrm{mm}$ scale; see Fig. 1 .

Rotational constants obtained from an estimated planar structure and dipole moments obtained from CNDO calculations suggested a spectrum mainly composed of $R$-branch transitions active through the $\mu_{\mathrm{a}}$ dipole moment; see Fig. 2.

We expected to find three nearly equidistant a-type $R$-lines with $\mathrm{K}_{-1}=0 \rightarrow 0$. Once these were found it was easy to determine the positions of the corresponding transitions $K_{-1}=1 \rightarrow 1$ and $K_{-1}=2 \rightarrow 2$; see Fig. 1 .

However, it is evident from comparison with the observed spectrum that the $R_{\mathrm{a}}$-transitions only form a part of this spectrum. The remaining spectral lines were indentified as $Q$ - and $R$-type transitions active through the $\mu_{\mathrm{b}}$ dipole moment. From this it is apparent that the $\mu_{\mathrm{a}}$ and $\mu_{\mathrm{b}}$ components of the dipole moment are of nearly equal magnitude, contrary to what was expected from the CNDO calculations.

The spectra of the two isotopic species $\mathrm{CH}_{2} \mathrm{C}^{35} \mathrm{ClCCH}$ and $\mathrm{CH}_{2} \mathrm{C}^{37} \mathrm{ClCCH}$ were distinguished and the strongest transitions were measured with high resolution; see Tables 1 and 2.

The rotational constants and five centrifugal distortion parameters were fitted to the observed spectrum by the least squares method; see Tables 3 and 4.

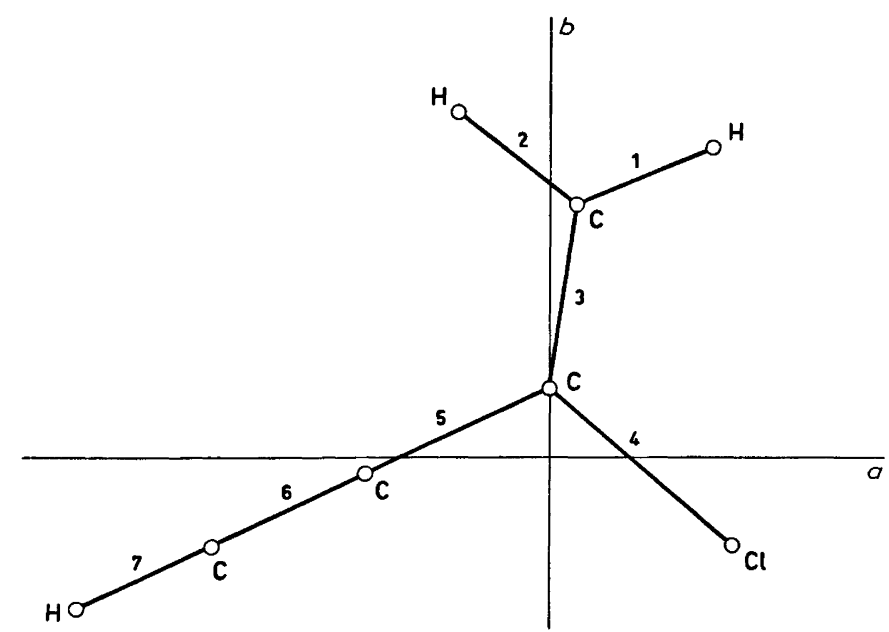

Fig. 2. Assumed structure of 2-chlorobuten-3-yne with principal axes of inertia.

Acta Chem. Scand. A 28 (1974) No. 2 
Table 1. The observed rotational transitions for 2-chlorobuten-3-yne, $\mathrm{CH}_{2} \mathrm{C}^{35} \mathrm{ClCCH}$, are given as $\nu_{\mathrm{obs}}=$ $v_{\text {center }}+\Delta v_{\mathrm{j}}(\mathrm{MHz})$ where $v_{\text {center }}$ is the center of the hypothetical unsplit line and $\Delta v_{1}, \Delta v_{2}, \Delta v_{3}$, and $\Delta v_{4}$ are the observed nuclear quadrupole hyperfine splitting of the rotational transition.

\begin{tabular}{|c|c|c|c|c|c|c|c|c|c|c|c|}
\hline $\begin{array}{l}\mathrm{CH}_{2} \\
J^{\prime}\end{array}$ & $\mathrm{CH}^{\mathrm{CH}^{35} \mathrm{Cl}}$ & $\stackrel{\mathrm{CCH}}{K_{+1}^{\prime}}$ & $J$ & $K_{-1}$ & $K_{+1}$ & $\mu$ & $\begin{array}{l}v_{\text {center }} \\
J_{K_{-1}^{\prime}}^{\prime} K_{+1}{ }^{\prime} \leftarrow K_{-1} K_{+1}\end{array}$ & $\stackrel{\Delta v_{1}}{F=} J+\frac{3}{2}$ & $\frac{\Delta v_{2}}{F=J+\frac{1}{2}}$ & $\frac{\Delta v_{3}}{F=J-\frac{1}{2}}$ & $\begin{array}{l}\Delta v_{4} \\
F=J-\frac{3}{2}\end{array}$ \\
\hline 6 & I & 6 & 5 & 1 & 5 & $\mathbf{a}$ & 27571.78 & 0.45 & -0.06 & -0.52 & -0.06 \\
\hline 5 & 1 & 4 & 4 & 1 & 3 & $\mathbf{a}$ & 27655.80 & 0.60 & -0.60 & -0.60 & 0.60 \\
\hline 6 & 0 & 6 & 5 & 0 & 5 & $a$ & 27908.99 & 0.55 & -0.33 & -0.55 & 0.22 \\
\hline 5 & 2 & 3 & 4 & 2 & 2 & $\mathbf{a}$ & 28170.07 & 0.28 & -0.28 & -0.28 & 0.28 \\
\hline 6 & 2 & 5 & 5 & 2 & 4 & $\mathbf{a}$ & 30653.63 & 0.62 & -0.63 & -0.63 & 0.62 \\
\hline 6 & $\mathbf{3}$ & 4 & 5 & 3 & 3 & $\mathbf{a}$ & 31863.50 & 0.67 & -0.99 & -0.65 & 1.06 \\
\hline 6 & 4 & 3 & 5 & 4 & 2 & $a$ & 31904.40 & 1.05 & -1.81 & -0.95 & 1.91 \\
\hline 7 & 1 & 7 & 6 & 1 & 6 & $\mathbf{a}$ & 31911.89 & 0.35 & -0.03 & -0.41 & -0.03 \\
\hline 6 & 4 & 2 & 5 & 4 & 1 & $a$ & 31957.64 & 1.01 & -1.75 & -0.88 & 1.83 \\
\hline 7 & 0 & 7 & 6 & 0 & 6 & $a$ & 32093.08 & 0.40 & -0.18 & -0.45 & 0.15 \\
\hline 6 & 1 & 5 & 5 & 1 & 4 & $a$ & 32515.04 & 0.64 & -0.65 & -0.65 & 0.64 \\
\hline 6 & 3 & 3 & 5 & 3 & 2 & $\mathbf{a}$ & 32704.69 & 0.36 & -0.55 & -0.36 & 0.64 \\
\hline 6 & 2 & 4 & 5 & 2 & 3 & $\mathbf{a}$ & 34060.29 & 0.21 & -0.21 & -0.21 & 0.21 \\
\hline 7 & 2 & 6 & 6 & 2 & 5 & $\mathbf{a}$ & 35393.24 & 0.48 & -0.49 & -0.49 & 0.48 \\
\hline 8 & 1 & 8 & 7 & 1 & 7 & $\mathbf{a}$ & 36216.51 & 0.28 & -0.03 & -0.29 & -0.03 \\
\hline 8 & 0 & 8 & 7 & 0 & 7 & $\mathbf{a}$ & 36307.46 & & & & \\
\hline 7 & 1 & 6 & 6 & 1 & 5 & $\mathbf{a}$ & 37005.51 & 0.67 & -0.67 & -0.67 & 0.67 \\
\hline 7 & 3 & 5 & 6 & 3 & 4 & $\mathbf{a}$ & 37118.84 & 0.57 & -0.57 & -0.57 & 0.57 \\
\hline 7 & 4 & 4 & 6 & 4 & 3 & $\mathbf{a}$ & 37341.30 & 0.70 & -1.13 & -0.65 & 0.95 \\
\hline 7 & 4 & 3 & 6 & 4 & 2 & $\mathbf{a}$ & 37512.02 & 0.61 & -0.99 & -0.48 & 0.71 \\
\hline 7 & 3 & 4 & 6 & 3 & 3 & $\mathbf{a}$ & 38768.29 & 0.09 & -0.10 & -0.10 & 0.09 \\
\hline 7 & 2 & 5 & 6 & 2 & 4 & $\mathbf{a}$ & 39725.92 & 0.27 & -0.27 & -0.27 & 0.27 \\
\hline 6 & 1 & 6 & 5 & 0 & 5 & b & 28260.99 & 0.63 & -0.46 & -0.68 & 0.41 \\
\hline 7 & 0 & 7 & 6 & 1 & 6 & b & 31741.10 & 0.31 & -0.04 & -0.34 & -0.04 \\
\hline 7 & 1 & 7 & 6 & 0 & 6 & b & 32263.90 & 0.44 & -0.32 & -0.51 & 0.18 \\
\hline 8 & 0 & 8 & 7 & 1 & 7 & $\mathbf{b}$ & 36136.59 & & & & \\
\hline 8 & 1 & 8 & 7 & 0 & 7 & $\mathbf{b}$ & 36387.33 & & & & \\
\hline 16 & 5 & 11 & 16 & 4 & 12 & $\mathbf{b}$ & 27367.92 & -0.73 & 0.72 & 0.72 & -0.73 \\
\hline 13 & 5 & 8 & 13 & 4 & 9 & $\mathbf{b}$ & 27690.70 & 0.19 & -0.20 & -0.20 & 0.19 \\
\hline 12 & 5 & 7 & 12 & 4 & 8 & b & 29903.39 & 0.30 & -0.30 & -0.30 & 0.30 \\
\hline 17 & 5 & 12 & 17 & 4 & 13 & $\mathbf{b}$ & 30384.34 & -1.09 & 1.09 & 1.09 & -1.09 \\
\hline 18 & 6 & 12 & 18 & 5 & 13 & $\mathbf{b}$ & 30684.75 & -0.15 & 0.15 & 0.15 & -0.15 \\
\hline 19 & 6 & 13 & 19 & $\mathbf{5}$ & 14 & $\mathbf{b}$ & 31375.11 & -0.49 & 0.49 & 0.49 & -0.49 \\
\hline 17 & 6 & 11 & 17 & 5 & 12 & $\mathbf{b}$ & 31609.18 & 0.09 & -0.09 & -0.09 & 0.09 \\
\hline 11 & 5 & 6 & 11 & 4 & 7 & b & 32293.77 & 0.19 & -0.19 & -0.19 & 0.19 \\
\hline 16 & 6 & 10 & 16 & 5 & 11 & b & 33741.40 & 0.31 & -0.32 & -0.32 & 0.31 \\
\hline 20 & 6 & 14 & 20 & 5 & 15 & b & 33864.55 & -0.80 & 0.79 & 0.79 & -0.80 \\
\hline 10 & 5 & 5 & 10 & 4 & 6 & $\mathbf{b}$ & 34379.12 & & & & \\
\hline 18 & 5 & 13 & 18 & 4 & 14 & $\mathbf{b}$ & 34934.45 & -1.33 & 1.32 & 1.32 & -1.33 \\
\hline 22 & 7 & 15 & 22 & 6 & 16 & $\mathbf{b}$ & 35522.62 & -0.31 & 0.30 & 0.30 & -0.31 \\
\hline 21 & 7 & 14 & 21 & 6 & 15 & b & 35564.87 & & & & \\
\hline 15 & 6 & 9 & 15 & 5 & 10 & b & 36504.02 & 0.36 & -0.36 & -0.36 & 0.36 \\
\hline 20 & 7 & 13 & 20 & 6 & 14 & $\mathbf{b}$ & 37227.33 & 0.23 & -0.22 & -0.22 & 0.23 \\
\hline 23 & 7 & 16 & 23 & 7 & 17 & $\mathrm{~b}$ & 37377.10 & -0.62 & 0.61 & 0.61 & 0.62 \\
\hline 21 & 6 & 15 & 21 & 5 & 16 & $\mathrm{~b}$ & 38088.53 & -1.08 & 1.07 & 1.07 & -1.08 \\
\hline 12 & 5 & 8 & 12 & 4 & 9 & $\mathrm{~b}$ & 38443.08 & -0.88 & 0.87 & 0.87 & -0.88 \\
\hline 14 & 6 & 8 & 14 & 5 & 9 & b & 39290.12 & 0.27 & -0.28 & -0.28 & 0.27 \\
\hline 13 & 5 & 9 & 13 & 4 & 10 & b & 39601.23 & -0.93 & 0.92 & 0.92 & -0.93 \\
\hline 25 & 8 & 17 & 25 & 7 & 18 & $\mathbf{b}$ & 39875.31 & -0.12 & 0.12 & 0.12 & -0.12 \\
\hline
\end{tabular}


Table 2. The observed rotational transitions for 2-chlorobuten-3-yne, $\mathrm{CH}_{2} \mathrm{C}^{37} \mathrm{ClCCH}$, given as in Table 1 .

\begin{tabular}{|c|c|c|c|c|c|c|c|c|c|c|c|}
\hline $\begin{array}{l}\mathrm{CH} \\
\boldsymbol{J}^{\prime}\end{array}$ & ${ }^{\mathrm{CH}_{-1}^{37}}$ & ${ }_{K_{+1}}^{\prime}$ & $\boldsymbol{J}$ & $K_{-1}$ & $K_{+1}$ & $\mu$ & $\begin{array}{l}v_{\text {center }} \\
J^{\prime} K_{-1}^{\prime} K_{+1}{ }^{\prime} \leftarrow J_{K_{-1}} K_{+1}\end{array}$ & $\begin{array}{l}\Delta v_{1} \\
F=J+\frac{s}{2}\end{array}$ & $\stackrel{\Delta v_{2}}{F=J+\frac{1}{2}}$ & $\begin{array}{l}\Delta v_{3} \\
F=J-\frac{1}{2}\end{array}$ & $\begin{array}{l}\Delta v_{4} \\
F=J-\frac{3}{2}\end{array}$ \\
\hline 6 & 1 & 6 & 5 & 1 & 5 & $\mathbf{a}$ & 27097.12 & 0.36 & -0.06 & -0.36 & -0.06 \\
\hline 6 & 0 & 6 & 5 & 0 & 5 & $a$ & 27441.68 & 0.45 & -0.23 & -0.48 & 0.15 \\
\hline 6 & 2 & 5 & 5 & 2 & 4 & $\mathbf{a}$ & 30098.45 & 0.48 & -0.48 & -0.48 & 0.48 \\
\hline 7 & $\overline{1}$ & 7 & 6 & $\overline{1}$ & 6 & $a$ & 31365.91 & 0.26 & -0.02 & -0.32 & -0.02 \\
\hline 6 & 1 & 5 & 5 & 1 & 4 & $a$ & 31938.57 & 0.44 & -0.44 & -0.44 & 0.44 \\
\hline 6 & 2 & 4 & 5 & 2 & $\mathbf{3}$ & $\mathbf{a}$ & 33388.35 & 0.18 & -0.19 & -0.19 & 0.18 \\
\hline 7 & 2 & 6 & 6 & 2 & 5 & $\mathbf{a}$ & 34761.56 & 0.39 & -0.39 & -0.39 & 0.39 \\
\hline 8 & 0 & 8 & 7 & 0 & 7 & $a$ & 35694.64 & 0.10 & -0.10 & -0.10 & 0.10 \\
\hline 7 & 1 & 6 & 6 & 1 & 5 & $a$ & 36373.95 & 0.50 & -0.51 & -0.51 & 0.50 \\
\hline 8 & 2 & 7 & 7 & 2 & 6 & $a$ & 39300.53 & 0.32 & -0.33 & -0.33 & 0.32 \\
\hline 9 & $\overline{1}$ & 9 & 8 & $\overline{1}$ & 8 & $\mathbf{a}$ & 39811.86 & & & & \\
\hline 9 & 0 & 9 & 8 & 0 & 8 & $\mathbf{a}$ & 39858.00 & & & & \\
\hline 7 & 0 & 7 & 6 & 1 & 6 & $\mathrm{~b}$ & 31185.01 & 0.22 & -0.02 & -0.03 & -0.02 \\
\hline 7 & 1 & 7 & 6 & 0 & 6 & $\mathbf{b}$ & 31734.44 & 0.38 & -0.38 & -0.38 & 0.38 \\
\hline 8 & 0 & 8 & 7 & 1 & 7 & $\mathbf{b}$ & 35513.67 & & & & \\
\hline 8 & 1 & 8 & 7 & 0 & 7 & b & 35780.23 & 0.20 & -0.20 & -0.20 & 0.20 \\
\hline 9 & 0 & 9 & 8 & 1 & 8 & $\mathbf{b}$ & 39772.35 & & & & \\
\hline 9 & 1 & 9 & 8 & 0 & 8 & b & 39897.53 & & & & \\
\hline 13 & 5 & 8 & 13 & 4 & 9 & b & 27850.47 & 0.07 & -0.08 & -0.08 & 0.07 \\
\hline 17 & 5 & 12 & 17 & 4 & 13 & b & 29563.19 & -0.75 & 0.74 & 0.74 & -0.75 \\
\hline 18 & 6 & 12 & 18 & 5 & 13 & $\mathbf{b}$ & 30615.62 & & & & \\
\hline 19 & 6 & 13 & 19 & 5 & 14 & b & 30950.69 & -0.34 & 0.34 & 0.34 & -0.34 \\
\hline 17 & 6 & 11 & 17 & $\mathbf{5}$ & 12 & $\mathbf{b}$ & 31790.55 & 0.06 & -0.07 & -0.07 & 0.06 \\
\hline 20 & 6 & 14 & 20 & $\mathbf{5}$ & 15 & $\mathbf{b}$ & 33018.42 & -0.59 & 0.58 & 0.58 & -0.59 \\
\hline 18 & 5 & 13 & 18 & 4 & 14 & $\mathbf{b}$ & 33772.03 & -0.95 & 0.94 & 0.94 & -0.95 \\
\hline 16 & 6 & 10 & 16 & 5 & 11 & $\mathbf{b}$ & 34045.91 & 0.22 & -0.22 & -0.22 & 0.22 \\
\hline 22 & 7 & 15 & 22 & 6 & 16 & $\mathbf{b}$ & 35213.51 & -0.17 & 0.17 & 0.17 & -0.17 \\
\hline 23 & 7 & 16 & 23 & 6 & 17 & $\mathbf{b}$ & 36567.66 & -0.41 & 0.40 & 0.40 & -0.41 \\
\hline 15 & 6 & 9 & 15 & 5 & 10 & $\mathbf{b}$ & 36807.87 & 0.21 & -0.22 & -0.22 & 0.21 \\
\hline 21 & 6 & 15 & 21 & 5 & 16 & b & 36810.64 & -0.76 & 0.76 & 0.76 & -0.76 \\
\hline 20 & 7 & 13 & 20 & 6 & 14 & b & 37594.53 & 0.16 & -0.16 & -0.16 & 0.16 \\
\hline 14 & 6 & 8 & 14 & 5 & 9 & b & 39505.29 & 0.11 & -0.11 & -0.11 & 0.11 \\
\hline 25 & 8 & 17 & 25 & 7 & 18 & $\mathbf{b}$ & 39747.82 & & & & \\
\hline
\end{tabular}

Table 3. Observed rotational constants in $\mathrm{MHz}$ and asymmetry parameters for the two isotopic species of 2-chlorobuten-3-yne.

\section{$\mathrm{CH}_{2} \mathrm{C}^{85} \mathrm{ClCCH} \quad \mathrm{CH}_{2} \mathrm{C}^{37} \mathrm{ClCCH}$}

\begin{tabular}{lcc}
\hline & & \\
$A$ & $6875.239 \pm 0.004$ & $6804.974 \pm 0.006$ \\
$B$ & $3096.353 \pm 0.002$ & $3034.415 \pm 0.003$ \\
$C$ & $2131.950 \pm 0.002$ & $2095.763 \pm 0.003$ \\
$\varkappa$ & -0.593 & -0.601 \\
\hline
\end{tabular}

\section{QUADRUPOLE COUPLING}

Because of the nuclear quadrupole coupling each rotational transition is split into four observable peaks with $\Delta F=+1$ for $R$-transitions and $\Delta F=0$ for $Q$-transitions. ${ }^{8}$ However, in many cases the components overlap partially or completely so that only a doublet or a single line can be distinguished.

We have used the splitting into quartets and doublets to obtain a least squares fitting of the two quadrupole coupling constants $\chi_{\mathrm{aa}}$ and $\chi_{\mathrm{bb}}$. For a doublet with line positions $\nu_{1}$ and $\nu_{2}$ the observed splitting used in the least squares fitting was $\Delta v_{Q} \mathrm{obs}=v_{2}-v_{1}$, and for a quartet $v_{1}, v_{2}, v_{3}$, and $v_{4}$ the observed splittings used were $\Delta v_{Q} \mathrm{obs}=\left(v_{4}+v_{3}-v_{2}-v_{1}\right) / 2$ and $\Delta v_{Q} \mathrm{Obs}=\left(v_{4}-v_{3}+v_{2}-v_{1}\right) / 2$.

If two lines are lying too close they may disturb each others line shape. Therefore in order to avoid systematic errors we only accepted splitting values $\Delta v_{Q}$ obs $>0.70 \mathrm{MHz}$. The error square sum is

$$
U=\sum\left(\Delta v_{Q} \text { obs }-\Delta v_{Q} \text { calc }\right)^{2}
$$

Acta Chem. Scand. A 28 (1974) No. 2 
Table 4. Measured and calculated centrifugal distortion constants in $\mathrm{kHz}$ for the two isotopic species of 2-chlorobuten-3-yne.

\begin{tabular}{|c|c|c|c|c|c|c|}
\hline \multirow{2}{*}{$\Delta_{\mathrm{J}}$} & \multicolumn{2}{|c|}{$\begin{array}{l}\mathrm{CH}_{2} \mathrm{C}^{35} \mathrm{ClCCH} \\
\text { obs }\end{array}$} & \multirow{2}{*}{$\begin{aligned} \text { calc } \\
0.89\end{aligned}$} & \multicolumn{2}{|c|}{$\begin{array}{l}\mathrm{CH}_{2} \mathrm{C}^{37} \mathrm{ClCCH} \\
\text { obs }\end{array}$} & \multirow{3}{*}{$\begin{array}{l}\text { calc } \\
0.87 \\
\quad 01\end{array}$} \\
\hline & 1.05 & \pm 0.02 & & 1.00 & \pm 0.02 & \\
\hline$\Delta_{\mathrm{IK}}$ & $-\mathbf{3 . 0 8}$ & \pm 0.05 & -1.60 & -3.46 & \pm 0.10 & \\
\hline$\Delta_{\mathrm{K}}$ & 16.0 & \pm 0.2 & 12.0 & 16.6 & \pm 0.3 & 12.4 \\
\hline$\delta_{\mathrm{I}}^{\mathbf{n}}$ & 0.427 & \pm 0.003 & 0.349 & 0.425 & \pm 0.006 & 0.344 \\
\hline$\delta_{\mathrm{K}}^{J}$ & 2.39 & \pm 0.05 & 2.19 & 2.17 & \pm 0.10 & 2.05 \\
\hline
\end{tabular}

It is possible to get rather good estimated values of $\chi_{\mathrm{aa}}{ }^{\prime}$ and $\chi_{\mathrm{bb}}{ }^{\prime}$ by comparing 2 . chlorobuten-3-yne with vinylchloride. ${ }^{9}$ The difference between the true values and the estimated values are

$\Delta \chi_{\mathrm{aa}}=\chi_{\mathrm{aa}}-\chi_{\mathrm{aa}}{ }^{\prime}$

$\Delta v_{\mathrm{bb}}=\chi_{\mathrm{bb}}-\chi_{\mathrm{bb}}{ }^{\prime}$

If $\Delta \chi_{\mathrm{aa}}$ and $\Delta \chi_{\mathrm{bb}}$ are assumed to be small it is possible to use the LETAGROP method to make the nonlinear least square fitting. ${ }^{10}$ According to this method, $U$ is regarded as a function of $\Delta \chi_{\mathrm{aa}}$ and $\Delta \chi_{\mathrm{bb}}$.

$U=U\left(\Delta \chi_{\mathrm{aa}}, \Delta \chi_{\mathrm{bb}}\right)$

The function $U$ is approximated by a second degree surface in the vicinity of $\chi_{\mathrm{aa}}{ }^{\prime}$ and $\chi_{\mathrm{bb}}{ }^{\prime}$.

$U=U^{\prime}+C_{1} \Delta \chi_{\mathrm{aa}}{ }^{2}+C_{2} \Delta \chi_{\mathrm{bb}}{ }^{2}+C_{3} \Delta \chi_{\mathrm{aa}} \Delta \chi_{\mathrm{bb}}+$

$+C_{4} \Delta \chi_{\mathrm{aa}}+C_{\mathrm{5}} \Delta \chi_{\mathrm{bb}}$

where $C_{1}, C_{2}, C_{3}, C_{4}$, and $C_{5}$ are constants and $U^{\prime}$ is the calculated error square sum for the estimated values

$\chi_{\mathrm{aa}}{ }^{\prime}$ and $\chi_{\mathrm{bb}}{ }^{\prime}$.

The improvements sought, $\Delta \chi_{\mathrm{aa}}$ and $\Delta \chi_{\mathrm{bb}}$, will give a minimum value for $U$. That is

$$
\begin{aligned}
& \partial U / \partial \Delta \chi_{\mathrm{aa}}=2 C_{1} \Delta \chi_{\mathrm{aa}}+C_{3} \Delta \chi_{\mathrm{bb}}+C_{4}=0 \\
& \partial U / \partial \Delta \chi_{\mathrm{bb}}=2 C_{2} \Delta \chi_{\mathrm{bb}}+C_{3} \Delta \chi_{\mathrm{aa}}+C_{5}=0
\end{aligned}
$$

These are the well known normal equations according to Gauss. The improvements $\Delta \chi_{\mathrm{aa}}$ and $\Delta \chi_{\mathrm{bb}}$ are obtained as solutions to these equations.

However, to be able to solve the normal equations the constants $C_{1}, C_{2}, C_{3}, C_{4}$, and $C_{5}$ have to be known. These constants could be determined if a sufficient set of trial values
$\Delta \chi_{\mathrm{aa}}$ and $\Delta \chi_{\mathrm{bb}}$ is chosen and inserted in the expression 4.

The method could be iterated and the convergence tested in the same way as the Gauss least square method. ${ }^{11}$ The LETAGROP method converges as rapid as the Gauss method. The great advantage is the simple way of obtaining the normal equations, but the number of computational operations increases rapidly with the number of constants sought.11

The values obtained are for ${ }^{85} \mathrm{Cl}$ in $\mathrm{CH}_{2} \mathrm{C}^{35}$. ClCCH: $\chi_{\mathrm{aa}}=-27.1 \pm 0.1$ and $\chi_{\mathrm{bb}}=-6.4 \pm 0.1$ $\mathrm{MHz}$ and for ${ }^{37} \mathrm{Cl}$ in $\mathrm{CH}_{2} \mathrm{C}^{37} \mathrm{ClCCH}$ : $\chi_{\mathrm{aa}}=$ $-21.8 \pm 0.3$ and $\chi_{\mathrm{bb}}=-4.0 \pm 0.3 \mathrm{MHz}$.

It is presently not possible to transform the quadrupole coupling constants from the inertial system to the principal axis system of the quadrupole tensor, since the angle between the $\mathrm{C}-\mathrm{Cl}$ bond and the principal axis $a$ is not accurately known; see Fig. 2.

\section{CENTRIFUGAL DISTORTION}

It was necessary to include the centrifugal distortion in the least square fitting process of the rotational constants. We used the five distortion parameters $\Delta_{\mathrm{J}}, \Delta_{\mathrm{JK}}, \Delta_{\mathrm{K}}, \delta_{\mathrm{J}}$ and $\delta_{\mathrm{K}}$ according to Watson. ${ }^{12}$ The 2-chlorobuten-3-yne molecule is planar but as it was possible to obtain a good fit with all five distortion constants we did not invoke the planarity constraints for these constants; ${ }^{13}$ see Table 4.

If the force field and geometry of a molecule is known it is possible to calculate the centrifugal distortion constants. ${ }^{8,14} \mathrm{We}$ have used a simple diagonal valence force field with force constants estimated from similar bonds in simpler molecules. ${ }^{15}$

The following symbols are used for the force constants according to Gribov. ${ }^{15} K_{\mathrm{i}}$ is the force 
constant for the stretching of the bond $i$, $K_{\mathrm{ij}}$ is the bending of the angle between the bonds $\mathrm{i}$ and $\mathrm{j}, l_{\mathrm{kl}} \mathrm{ij}$ is the interaction between the bending $K_{\mathrm{ij}}$ and $K_{\mathrm{kl}}, \varrho_{\mathrm{k}} \mathrm{ij}$ is the bending of the bond $\mathrm{k}$ out of the plane spanned by the bonds $i$ and $j, \chi_{k 1}{ }^{i j}$ finally is the torsion between the planes spanned by the bonds $i$ and $\mathrm{j}$, and $\mathrm{k}$ and $\mathrm{l}$, respectively.

The values of the constants are, with the bond numbers of Fig. 2:

$K_{1}=K_{2}=5.53, \quad K_{3}=8,44 \quad K_{4}=3.85, \quad K_{5}=3.77$, $K_{6}=13.33, K_{7}=5.99, \quad K_{12}-2 l_{13}{ }^{12}+l_{23}{ }^{13}=0.42$, $K_{13}-l_{23}{ }^{13}=0.59, \quad K_{34}-l_{34}{ }^{35}-l_{34}{ }^{45}+l_{35}{ }^{45}=0.84$, $K_{35}-l_{34}{ }^{35}+l_{34}{ }^{45}-l_{35}{ }^{45}=0.84, \quad K_{45}+l_{34}{ }^{35}-l_{34}{ }^{45}-$ $l_{35}{ }^{45}=1.03, \quad K_{56}=0.32, \quad K_{67}=0.23, \quad \varrho_{3}{ }^{12}=0.224$, $\varrho_{3}{ }^{45}=0.406$ and $\chi_{45}{ }^{12}=0.512$ in $\mathrm{md} / \AA$ and md $\AA$ for stretching and bending constants, respectively.

We have assumed the same force constants for bending in plane and out of plane for the linear acetylenic group.

The calculated fundamental frequencies were compared with the observed IR-spectrum but we have not been able to make a complete assignment yet. However, some of the eighteen fundamentals are readily observed. For a planar asymmetric rotor theoretical calculations give four independent distortion constants $\tau_{\text {aaaa }}, \tau_{\mathrm{bbbb}}, \tau_{\mathrm{aabb}}$ and $\tau_{\mathrm{abab}}{ }^{8}$

The centrifugal distortion parameters according to Watson could be obtained from these constants if the rotational constants are known; ${ }^{12}$ see Table 4. The agreement between measured and calculated values is rather good for all constants but $\Delta_{\mathrm{JK}}$. However, the constant $\Delta_{\mathrm{JK}}$ seems to be extremely sensitive to the bending force constants $K_{34}, K_{35}$ and $K_{45}$, and it has been rather difficult to obtain a good estimation of it.

\section{MOLECULAR STRUCTURE}

The rotational constants $A, B$, and $C$ are accurately obtained from the measured spectrum since both $R$ - and $Q$-type transitions have been observed. Therefore we could expect the derived principal moments of inertia $I_{\mathrm{a}}{ }^{0}, I_{\mathrm{b}}{ }^{0}$ and $I_{\mathrm{c}}{ }^{0}$ to be accurate. These are structure dependent parameters and could eventually be used as additional constraints in other structure determining methods, e.g. electron diffraction in gases. ${ }^{16}$ However, it is then necessary to transform the observed moments of inertia $I_{\mathrm{a}}{ }^{0}, I_{\mathrm{b}}{ }^{0}$ and $I_{\mathrm{c}}{ }^{0}$ for rotational spectroscopy to the moments of inertia $I_{\mathrm{a}}{ }^{\mathrm{z}}, I_{\mathrm{b}}{ }^{\mathrm{z}}$ and $I_{\mathrm{c}}{ }^{\mathrm{z}}$ for the average structure. ${ }^{2,17}$ This correction is given by

$I_{\alpha}{ }^{z}=I_{\alpha}{ }^{0}-\frac{1}{2} \sum \varepsilon_{\mathrm{s}}{ }^{\alpha}($ har $)+\delta I_{\alpha}$ (cent) $\alpha=\mathrm{a}$, b or c

for an asymmetric rotor, where $I_{\alpha}{ }^{0}$ is one of the three principal moments of inertia for the effective structure in the ground state, and $I_{\alpha}{ }^{z}$ is one of the three principal moments of inertia for the average structure. ${ }^{8}$ The sum is over all fundamental vibrations.

The vibration-rotation term $\varepsilon_{\mathbf{s}}{ }^{\alpha}($ har) depends on the harmonic potentials and is obtained from a normal coordinate analysis.

The centrifugal distortion term $I_{\alpha}$ (cent) is also obtainable from the force field and geometry. ${ }^{17}$ The effects of the corrections according to (6) is given in Table 5. Evidently the centrif-

Table 5. Vibration-rotation correction for the moments of inertia and inertial defect for the ground vibrational state (in au $\AA^{2}$ ). Conversion factor 505376 au $\AA^{2} \mathrm{MHz}$.

\begin{tabular}{|c|c|c|c|c|c|}
\hline & & $r_{0}$-parameters & $\begin{array}{l}\text { vibration } \\
\text { correction }\end{array}$ & $\begin{array}{l}\text { centrifugal } \\
\text { distortion } \\
\text { correction }\end{array}$ & $r_{z}$-parameters \\
\hline $\mathrm{C}_{4} \mathrm{H}_{3}{ }^{35} \mathrm{Cl}$ & $\begin{array}{l}I_{\mathrm{a}} \\
I_{\mathrm{b}} \\
I_{\mathrm{c}} \\
\Delta\end{array}$ & $\begin{array}{r}73.5066 \\
163.2165 \\
237.0487 \\
0.3256\end{array}$ & $\begin{array}{l}0.1270 \\
0.1884 \\
0.0063 \\
-\end{array}$ & $\begin{array}{r}0.0000 \\
0.0002 \\
-0.0007 \\
-\end{array}$ & $\begin{array}{r}73.6336 \\
163.4051 \\
237.0543 \\
0.0156\end{array}$ \\
\hline $\mathrm{C}_{4} \mathrm{H}_{3}{ }^{37} \mathrm{Cl}$ & $\begin{array}{l}I_{\mathrm{a}} \\
I_{\mathrm{b}} \\
I_{\mathrm{c}} \\
\Delta\end{array}$ & $\begin{array}{r}74.2657 \\
166.5481 \\
241.1418 \\
0.3280\end{array}$ & $\begin{array}{l}0.1271 \\
0.1919 \\
0.0078 \\
-\end{array}$ & $\begin{array}{r}0.0000 \\
0.0002 \\
-0.0007 \\
-\end{array}$ & $\begin{array}{r}74.3928 \\
166.7402 \\
241.1489 \\
0.0159\end{array}$ \\
\hline
\end{tabular}

Acta Chem. Scand. A 28 (1974) No. 2 
ugal distortion contribution can be neglected in this case.

It is also interesting to see that the $I_{\mathrm{c}}{ }^{0}$ constant is little affected by these corrections compared with $I_{\mathrm{a}}{ }^{0}$ and $I_{\mathrm{b}}{ }^{0}$.

The inertial defect

$\Delta=I_{\mathrm{c}}-I_{\mathrm{a}}-I_{\mathrm{b}}$

vanishes for an average planar structure as it would for a hypothetical stiff planar rotor. Thus the combination of (6) and (7) gives

$$
\begin{aligned}
& \Delta^{z}=\Delta^{0}-\frac{1}{2} \sum_{\mathrm{s}}\left(\varepsilon_{\mathrm{s}}{ }^{\mathrm{c}}-\varepsilon_{\mathrm{s}}{ }^{\mathrm{a}}-\varepsilon_{\mathrm{s}}{ }^{\mathrm{b}}\right)+\delta I_{\mathrm{c}}(\text { cent })- \\
& \delta I_{\mathrm{a}}(\text { cent })-\delta I_{\mathrm{b}}(\text { cent })=0
\end{aligned}
$$

where $\Delta^{z}$ and $\Delta^{0}$ are the inertial defect for the average and effective structure respectively. ${ }^{18,10}$ Therefore it is possible to calculate the inertial defect and to control the validity of the correction terms to some extent by comparing the measured and calculated inertial defects. See Table 5. The obtained deviation of $\Delta^{z}$ from zero is the difference between the measured and calculated inertial defects and this is found to be $\mathbf{0 . 0 1 6}$ for both isotopic species. This is quite satisfactory considering the simple estimated force field used.

Acknowledgements. We thank all those who have contributed to this paper and especially Mr. Hasse Karlsson. Dr. Stig Ljunggren, and Professor Gunnar Erlandsson for their generous cooperation.

\section{REFERENCES}

1. Karlsson, F. and Vestin, R. Acta Chem. Scand. 27 (1973) 3033.

2. Morino, Y., Kuchitsu, K. and Oka, T. J. Chem. Phys. 36 (1962) 1108.

3. Herschbach, D. R. and Laurie, V.W. $J$. Chem. Phys. 37 (1962) 1668.

4. Oka, T. J. Phys. Soc. Jap. 15 (1960) 2274.

5. Nieuwland, J. A. and Vogt, R. The Chemistry of Acetylene, New York 1945, p. 160.

6. Finlay, J. B. (du Pont) U. S. 2.999.887, 1961.

7. Vestin, R., Borg, A. and Lindblom, T. Acta Chem. Scand. 22 (1968) 687.

8. Gordy, W. and Cook, R. L. Microwave Molecular Spectra, Interscience, New York 1970.

9. Gerry, M. C. L. Can. J. Chem. 49 (1971) 255.

10. Sillén, L. G. Acta Chem. Scand. 16 (1962) 159.

11. Karlsson, F. and Vestin, R. Chem. Scr. 2 (1972) 207.
12. Watson, J. K. G. J. Chem. Phys. 46 (1967) 1935.

13. Kirchhoff, W.H. J. Mol. Spectrosc. 41 (1972) 333

14. Ramaswamy, K, and Srinivasan, K. J. Mol. Struct. 3 (1969) 473.

15. Gribov, L. A. Vvedenie $v$ teoriyu $i$ raschët kolebatel' nȳkh spektrov mnogoatomnȳkh molekul, Istadel'stvo Leningradskogo Universita, 1965.

16. Kuchitsu, K., Fukuyama, T. and Morino, Y. J. Mol. Struct. 1 (1968) 463.

17. Oka, T. and Morino, Y.J. Mol. Spectrosc. 8 (1962) 300 .

18. Darling, B. T. and Dennison, D. M. Phys. Rev. 57 (1940) 128.

19. Oka, T. and Morino, Y. J. Mol. Spectrosc. 6 (1961) 472 .

Received November 2, 1973. 